\title{
Synthesis of 6-chloro-2-(propargyloxy)quinoline-4-carboxylic acid and propargyl 6-chloro-2-(propargyloxy)quinoline-4-carboxylate
}

\section{R. Bouhfid, E.M. Essassi*}

Laboratoire de Chimie Organique Hétérocyclique, Université Mohammed V-Agdal, BP: 1014 Avenue Ibn Batouta, Rabat, Maroc.

E-mail: emessassi@yahoo.fr

*Author to whom correspondence should be addressed

Received: 10 February 2006 / Accepted: 20 March 2006 / Published: 1 September 2006

Keywords: quinoline, alkylation, esterification, propargyl bromide.

The quinoline ring systems are important structural units in naturally occurring alkaloids and synthetic analogues with interesting biological activities. Therefore, the development of new and efficient synthetic route for the preparation of their analogues is of importance in both synthetic organic chemistry and medicinal chemistry. ${ }^{1-4}$

We reported here the synthesis of a new quinoline derivative.

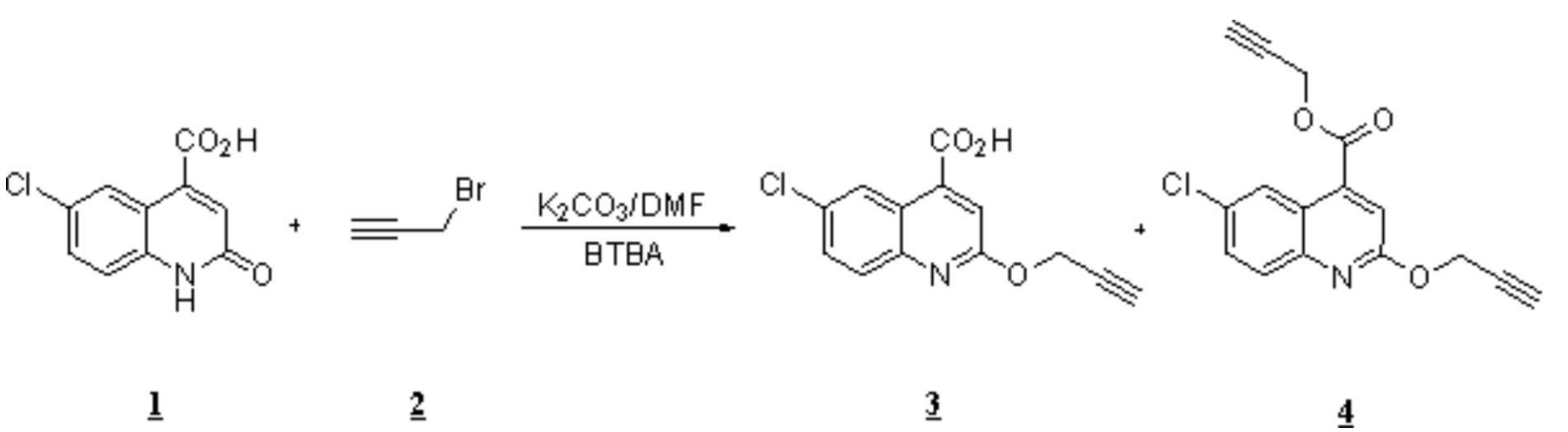

To a solution of quinoline $\underline{1}(1 \mathrm{~g}, 4.4 \mathrm{mmol})$ and $\mathrm{K}_{2} \mathrm{CO}_{3}(1.21 \mathrm{~g}, 8.8 \mathrm{mmol})$ in $60 \mathrm{~mL}$ of DMF, was added propargyl bromide $(0.75 \mathrm{~mL}, 8.8 \mathrm{mmol})$ and tetra n-butylammonium bromide (TBAB) (catalytic amount). The mixture was stirred at room temperature for $24 \mathrm{~h}$ and the reaction was quenched by the addition of saturated aqueous $\mathrm{NaHCO}_{3}$. The mixture was extracted with $\mathrm{Et}_{2} \mathrm{O}$ and the combined organic layers were washed with brine and dried over $\mathrm{Na}_{2} \mathrm{SO}_{4}$. After removal of the solvent, the residue was purified by column chromatography on silica gel (n-hexane/AcOEt 8:2) to give $0.44 \mathrm{~g}(43 \%)$ of $\underline{3}$ and $0.46 \mathrm{~g}(35 \%)$ of $\underline{4}$.

\section{6-chloro-2-(propargyloxy)quinoline-4-carboxylic acid, $\underline{3}$}

Melting point: $170^{\circ} \mathrm{C}$

${ }^{1} \mathrm{H}-\mathrm{NMR}\left(300 \mathrm{MHz}, \mathrm{CDCl}_{3}\right): \delta=2.62\left(\mathrm{t}, 1 \mathrm{H}, \equiv \mathrm{CH},{ }^{3} \mathrm{~J}=2.4 \mathrm{~Hz}\right) ; 5.09\left(\mathrm{~d}, 2 \mathrm{H}, \mathrm{OCH}_{2},{ }^{3} \mathrm{~J}=2.4 \mathrm{~Hz}\right) ; 7.61(\mathrm{~s}$, $1 \mathrm{H},=\mathrm{CH}) ; 7.64-8.53\left(\mathrm{~m}, 3 \mathrm{H}, \mathrm{H}_{\mathrm{Ar}}\right)$.

${ }^{13} \mathrm{C}-\mathrm{NMR}\left(300 \mathrm{MHz}, \mathrm{CDCl}_{3}\right): \delta=54.7\left(\mathrm{OCH}_{2}\right) ; 76.1(\equiv \mathrm{CH}) ; 118.1(=\mathrm{CH}) ; 127.1,129.6,133.7\left(\mathrm{CH}_{\mathrm{Ar}}\right)$; $120.1,137.3,143.8(\mathrm{Cq}) ; 164.0(\mathrm{C}=\mathrm{N}) ; 164.2\left(\mathrm{CO}_{2} \mathrm{H}\right)$.

$\operatorname{MS}(\mathrm{EI}, \mathrm{m} / \mathrm{z}): 237$.

Elemental analysis: Calculated for $\mathrm{C}_{13} \mathrm{H}_{8} \mathrm{ClNO}_{3}: \mathrm{C}, 59.67 \%$; H, $3.08 \%$; $5.35 \%$; Found: C, $59.70 \%$; H, $3.04 \%$; N, $5.41 \%$; 


\section{Propargyl-6-chloro-2-(propargyloxy)quinoline-4-carboxylate, $\underline{4}$}

Melting point: $156^{\circ} \mathrm{C}$

${ }^{1} \mathrm{H}-\mathrm{NMR}\left(300 \mathrm{MHz}, \mathrm{CDCl}_{3}\right): \delta=2.67\left(\mathrm{t}, 1 \mathrm{H}, \equiv \mathrm{CH},{ }^{3} \mathrm{~J}=2.4 \mathrm{~Hz}\right) ; 2.70\left(\mathrm{t}, 1 \mathrm{H}, \equiv \mathrm{CH},{ }^{3} \mathrm{~J}=2.4 \mathrm{~Hz}\right) ; 5.05(\mathrm{~d}$, $\left.2 \mathrm{H}, \mathrm{OCH}_{2},{ }^{3} \mathrm{~J}=2.4 \mathrm{~Hz}\right) ; 5.12\left(\mathrm{~d}, 2 \mathrm{H}, \mathrm{OCH}_{2},{ }^{3} \mathrm{~J}=2.4 \mathrm{~Hz}\right) ; 7.08(\mathrm{~s}, 1 \mathrm{H},=\mathrm{CH}) ; 7.58-8.20\left(\mathrm{~m}, 3 \mathrm{H}, \mathrm{H}_{\mathrm{Ar}}\right)$.

${ }^{13} \mathrm{C}-\mathrm{NMR}\left(300 \mathrm{MHz}, \mathrm{CDCl}_{3}\right): \delta=52.4\left(\mathrm{OCH}_{2}\right) ; 54.4\left(\mathrm{OCH}_{2}\right) ; 78.1(\equiv \mathrm{CH}) ; 78.7(\equiv \mathrm{CH}) ; 78.9,75.5(\equiv \mathrm{C})$; $118.2(=\mathrm{CH}) ; 125.5,126.4,131.9\left(\mathrm{CH}_{\mathrm{Ar}}\right) ; 118.5,138.1,138.2(\mathrm{Cq}) ; 159.7(\mathrm{C}=\mathrm{N}) ; 164.3\left(\mathrm{CO}_{2} \mathrm{H}\right)$.

MS (EI, m/z): 299.

Elemental analysis: Calculated for $\mathrm{C}_{16} \mathrm{H}_{10} \mathrm{ClNO}_{3}$ : C, $64.12 \%$; $3.36 \%$; N, $4.67 \%$; Found: $\mathrm{C}, 64.17 \%$; H, $3.29 \%$; N $4.72 \%$;

References:

1. Balasubramanian, M.;Keay, J. G. Pyridines and their Benzo Derivatives: Application In Comprehensive Heterocyclic Chemistry II;Katrizky, A. P., Rees, V. W., Scriven, E. F., Eds.;Pergamon: Oxford, 1996; Vol. 5, pp 245-300.

2. Ranu, B. C.; Hajra, A.; Dey, S. S.; Jana, U. Tetrahedron 2003, 59, 813-819.

3. Wada, Y.; Mori, T.; Ichikawa, J. Chem. Lett. 2003, 32, 1000-1001.

4. Kobayashi, K.; Yoneda, K.; Mizumoto, T.; Umakoshi, H.; Morikawa, O.; Konishi, H. Tetrahedron Lett. 2003, 44, 4733-4736.

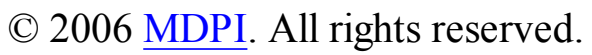

